\title{
Reflexive Symmetry Detection in Single Image
}

\author{
Zhongwei Tang ${ }^{1}$, Pascal Monasse $^{2}$, and Jean-Michel Morel ${ }^{1}$ \\ 1 CMLA-ENS Cachan, 61 avenue du Président Wilson, BP 94235 Cachan - France \\ \{zhongwei.tang, jean-michel.morel\}@cmla.ens-cachan.fr \\ 2 Université Paris-Est, LIGM (UMR CNRS 8049), ENPC, F-77455 Marne-la-Vallée \\ monasse@imagine.enpc.fr
}

\begin{abstract}
Reflective symmetry can be used as a strong prior for many computer vision tasks. We interpret the planar reflective symmetry detection by using the property of an involution, which implies that two pairs of matched points are enough to define a planar reflective symmetry observed from a non-frontal viewpoint. This makes the reflective symmetry estimation as efficient as the classical homography estimation in binocular stereovision. This simple reflective symmetry computation can be plugged into any multiple model estimation to detect multiple symmetries at different scales and locations in images. The experimental results show that the proposed method is able to detect single and multiple reflective symmetries both in frontal and non fronto-parallel viewpoints.
\end{abstract}

Keywords: reflective symmetry, perspective distortion, involution, multiple model estimation

\section{Introduction}

Reflective symmetry is very common in artificial environments, and also in the natural world, especially with living forms. For the semantic interpretation of a photograph, an accurate detection of the symmetries is a valuable mid-level input. Leveraging on reliable feature point detection and matching, such as SIFT [7], a few methods were proposed to detect reflective symmetries in images. For example, Loy and Eklundh [8] use a Hough transform approach to symmetry detection, where each pair of matching points contributes with a weight to a parameterized symmetry axis set. They show that SIFT descriptors can be directly manipulated to provide descriptors of the virtual reflected image. Though it may be faster than applying the full SIFT pipeline to the mirror image, we find it simpler to use the latter. A main limitation of the method is the restriction to fronto-parallel symmetry, since the symmetry axis is supposed to be the median line of the segment joining matching points. When the symmetric structure is viewed from a titled angle, this assumption does not hold and the algorithm is not applicable. A related approach is the one of Liu et al. [6], that tries a large set of possible symmetry lines and computes for each a matching score based on edge directions. 
One of the best performing methods is the one of Cho and Lee [1]. It finds regions of symmetry by growing from a matched pair based on photometric similarity and geometric consistency. Still, it maintains the assumption of frontoparallel view of the symmetric object.

Being able to detect symmetries without relying on the fronto-parallel view assumption is still a challenge. Though this assumption was lifted in some algorithms for detection of repetitions, such as the one of Tuytelaars et al. [11], to the best of our knowledge it is the first time a fully developped algorithm not relying on this assumption is presented.

From a single pair of matching points, the symmetry axis cannot be detected when the fronto-parallel hypothesis is not satisfied. However, we show that two matching pairs are enough to estimate it. This suggests an algorithm of type RANSAC [4] to recover a symmetry. In Section 2, we formulate the geometric problem, propose a parameterized representation of the symmetry and a simple method to estimate it, much akin to homography estimation from four point matches. An algorithm detecting the symmetry is then proposed. Extending it to the detection of multiple symmetries in the image is not straightforward. Section 3 shows that relying on the J-linkage algorithm [10] provides an efficient generalization to the detection of multiple symmetries in skewed views. Experiments on synthetic and real images and qualitatively and quantitatively evaluated in Section 4, showing that the proposed method establishes the state of the art in detection accuracy. Finally, some extensions are suggested in Section 5.

\section{Single Symmetry Detection}

\subsection{Problem statement}

Let us consider 2D points $P_{i}$ and $P_{i}^{\prime}$ that are image of each other under a planar symmetry. Assuming the points are represented by 3 -vectors in homogeneous coordinates $p_{i}$ and $p_{i}^{\prime}$, we can write

$$
p_{i}^{\prime}=T p_{i} \text { with } T=\left(\begin{array}{ccc}
-1 & 0 & 0 \\
0 & 1 & 0 \\
0 & 0 & 1
\end{array}\right) .
$$

The equalities have to be understood up to a scalar factor. If these points lie on a $2 \mathrm{D}$ plane viewed by a pinhole camera, the points undergo a projective transformation, represented by homography matrix $H$ [5]. Let $q_{i}=H p_{i}$ and $q_{i}^{\prime}=H p_{i}^{\prime}$ be the projections of $P_{i}$ and $P_{i}^{\prime}$ into the image plane. From (1), we deduce

$$
q_{i}^{\prime}=\hat{H} q_{i} \text { with } \hat{H}=H T H^{-1} .
$$

From the obvious equality $T^{2}=I_{3}$, the $3 \times 3$ identity matrix, we deduce the analogous equation for $\hat{H}$

$$
\hat{H}^{2}=I_{3},
$$

showing that $\hat{H}$, the $H$-conjugate of $T$, is still an involution. 
To estimate such an involution $\hat{H}$ from point correspondences $\left(q_{i}, q_{i}^{\prime}\right)$, we propose two methods, exposed in the following sections. The first one is based on a parameterization of an involution matrix and requires algebraic geometry techniques. The second one is simpler and direct, related to the standard 4-point homography estimation.

\subsection{Parameterized Involution Computation}

Notice that

$$
\left(\begin{array}{ll}
a & 0 \\
0 & B
\end{array}\right) T\left(\begin{array}{ll}
a & 0 \\
0 & B
\end{array}\right)^{-1}=T
$$

for any real $a \neq 0$ and $B$ a $2 \times 2$ invertible matrix. We can always choose $a$ and $B$ such that

$$
H\left(\begin{array}{cc}
a & 0 \\
0 & B_{2 \times 2}
\end{array}\right)=\left(\begin{array}{lll}
1 & x & y \\
z & 1 & 0 \\
t & 0 & 1
\end{array}\right):=\tilde{H}(x, y, z, t) \text { with } x z+y t \neq 1 .
$$

In effect, the equation above parameterizes an involution by four parameters $x$, $y, z$, and $t$. This yields

$$
\hat{H}=\tilde{H} T \tilde{H}^{-1}=\frac{1}{1-x z-y t}\left(\begin{array}{ccc}
1-x z-y t & 2 x & 2 y \\
-2 z & 1+x z-y t & 2 y z \\
-2 t & 2 x t & 1-x z+y t
\end{array}\right) .
$$

We see that from two pairs of corresponding points $\left(q_{i}, q_{i}^{\prime}\right), i=1,2,(2)$ results in four algebraic equations of degree 2 in the unknowns $(x, y, z, t)$. This can yield up to $2^{4}=16$ real solutions. These solutions can be obtained through Gröbner bases extraction for example [2]. To disambiguate these solutions, a third pair should be used. This method has the advantage that it parameterizes explicitly the unknown matrix $\hat{H}$, but the solution presented in the next section, while providing less insight, has the advantage of being much simpler to compute.

\subsection{Direct Involution Computation}

Instead of parameterizing explicitly an involution matrix $\hat{H}$, we can just write

$$
\left\{\begin{array}{l}
q_{i}^{\prime}=\hat{H} q_{i} \\
q_{i}=\hat{H} q_{i}^{\prime}
\end{array}\right.
$$

Equalities being up to a scalar factor, a pair of matching points yields 4 independent equations. Considering that $\hat{H}$ has 8 variables (a $3 \times 3$ matrix defined up to scale), two distinct pairs provide exactly one linear system for the coefficients of $\hat{H}$. This is very similar to the standard homography estimation from 4 point matches, namely here $\left(q_{1}, q_{1}^{\prime}\right),\left(q_{1}^{\prime}, q_{1}\right),\left(q_{2}, q_{2}^{\prime}\right)$, and $\left(q_{2}^{\prime}, q_{2}\right)$.

Notice that a geometric interpretation of the involution is quite simple. The line of symmetry $L$ can be parameterized by two points: 
1. The intersection of the lines $p_{1} \times p_{2}$ and $p_{1}^{\prime} \times p_{2}^{\prime}$.

2. The intersection of the diagonal $p_{1} \times p_{2}^{\prime}$ and $p_{2} \times p_{1}^{\prime}$.

The notation $\times$ corresponds to the cross-product of homogeneous vectors representing the points and gives the three coefficients of the line equation. Now, let $C$ be the intersection of the lines $p_{1} \times p_{1}^{\prime}$ and $p_{2} \times p_{2}^{\prime}$. This point is at infinity in the frontal view. The image $p_{3}^{\prime}$ of a point $p_{3}$ through the symmetry is obtained through the following process:

1. Let $D$ be the intersection of lines $L$ and $p_{1} \times p_{3}$.

2. The point $p_{3}^{\prime}$ is at the intersection of $C \times p_{3}$ and $D \times p_{1}^{\prime}$.

Of course, the above construction can be obtained also with $p_{2}$ and $p_{2}^{\prime}$.

The geometric construction described above is based only on line intersections. The consequence is that it can be translated directly in the non-frontal view through the homography $H$. This is illustrated in Figure 1.

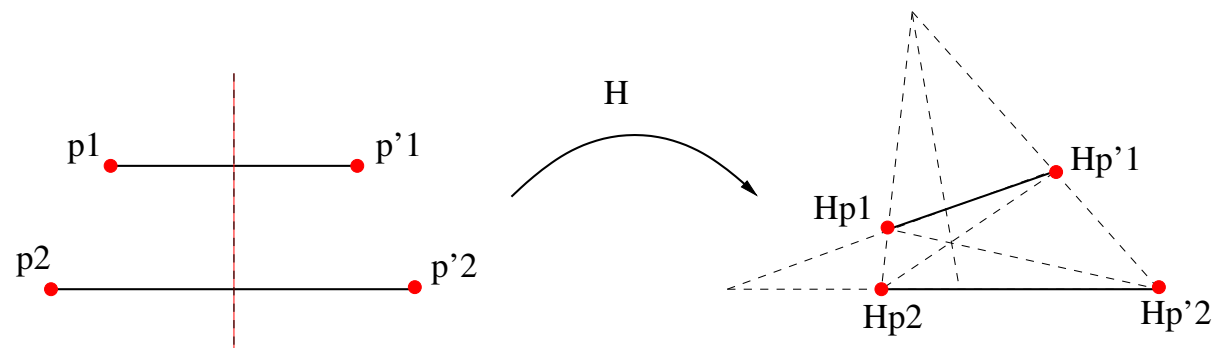

Fig. 1. Reflective symmetry through a homography. See text for the geometric construction illustrated in right figure.

\subsection{Algorithm}

The algorithm to detect a symmetry in the image is to use the RANSAC procedure [4] with the 2-correspondence algorithm presented in previous section. More precisely:

1. Compute reflected image $\tilde{I}(x, y)=I(w-x, y)$ of original image $I$ of width $w$.

2. Match SIFT keypoints in $I$ and $\tilde{I}:\left(q_{i}, q_{i}^{\prime}\right)_{i \in\{1, \cdots, n\}}$.

3. Run RANSAC using 2-correspondence solver:

- Take random pair $(i, j) \in\{1, \cdots, n\}$, and compute $H_{i, j}$.

- If \#\{k:d(H,j$\left.\left.q_{i}, q_{i}^{\prime}\right)<\sigma\right\}$ exceeds its current maximal value, update the best homography $H \leftarrow H_{i, j}$.

- If planned number of iterations is not yet reached, iterate. 
To make the process more robust, we can use the scale and orientation of SIFT descriptors to check a homography $H_{i, j}$ : after rectification, we must have for $k=i$ and $k=j$

$$
\begin{aligned}
\left|s\left(p_{k}\right)-s\left(p_{k}^{\prime}\right)\right| & <\delta \max \left(s\left(p_{k}\right), s\left(p_{k}^{\prime}\right)\right) \\
\cos \left(\theta_{k}+\theta_{k}^{\prime}\right) & <-1+\varepsilon
\end{aligned}
$$

where $s$ is the scale associated to keypoint and $\theta$ the orientation.

\section{Multiple Symmetries}

It is not a rare occurence to have multiple symmetries in images. The standard approaches to detect parameterized models are the Hough transform and the RANSAC algorithm. The first one has the problem of an efficient sampling of the parameter space and the second one is not naturally tuned to multiple model detection. An alternative combining the two approaches is the J-linkage algorithm [10]. It stores in a binary matrix $C$ the coherence between model and data. More precisely, $C(i, j)=1$ iff data index $i$ is compatible with model number $j$. A row $C(i,:)$ is the preference set (PS) of sample $i$ and a column $C(:, j)$ is the consensus set (CS) of model $j$. The J-linkage algorithm clusters the set of data points in a bottom-up procedure. Each point $i$ is initially in its own cluster $C_{i}=\{i\}$. The two clusters $\left(i, i^{\prime}\right)$ with minimal Jaccard distance are merged into cluster $C_{i} \cup C_{i^{\prime}}$ whose PS is the intersection of the two PSs of $i$ and $i^{\prime}$. This is iterated until the minimal Jaccard distance becomes 1 . The Jaccard distance is:

$$
d_{J}(A, B)=\frac{|A \cup B|-|A \cap B|}{|A \cup B|} .
$$

A model is adjusted to each final cluster by least square fitting to the data points.

The success of J-linkage relies crucially on a non-uniform sampling of the models, contrary to RANSAC. The reason is that if true models are oversampled, we get stable row features (PS). We use the following sampling strategy to select pairs of matching points that generate a model:

$$
\begin{aligned}
& \mathbb{P}\left(m_{i}\right)=\frac{1}{Z_{1}} \exp \left(-\frac{1}{\sigma_{d}^{2}}\left(\left\|\operatorname{SIFT}\left(q_{i}\right)-\operatorname{SIFT}\left(q_{i}^{\prime}\right)\right\|-d_{0}\right)^{2}-\frac{1}{\sigma_{l}^{2}}\left(\left\|q_{i}-q_{i}^{\prime}\right\|-l_{0}\right)^{2}\right) \\
& \mathbb{P}\left(m_{j} \mid m_{i}\right)=\frac{1}{Z_{2}} \exp \left(-\frac{1}{\sigma_{c}^{2}}\left(\left\|c_{i}-c_{j}\right\|-c_{0}\right)^{2}\right),
\end{aligned}
$$


where $m_{i}=\left(q_{i}, q_{i}^{\prime}\right) . Z_{1}$ and $Z_{2}$ are normalization factors. The parameters are estimated based on statistics of distances:

$$
\begin{aligned}
d_{0} & =\frac{1}{2 N} \sum_{m_{i}}\left\|\operatorname{SIFT}\left(q_{i}\right)-\operatorname{SIFT}\left(q_{i}^{\prime}\right)\right\| \\
\sigma_{d}^{2} & =\frac{1}{10} \max _{m_{i}}\left\|\operatorname{SIFT}\left(q_{i}\right)-\operatorname{SIFT}\left(q_{i}^{\prime}\right)\right\|^{2} \\
l_{o} & =\sqrt{w^{2}+h^{2}} \\
\sigma_{l}^{2} & =\frac{1}{10} \max _{m_{i}}\left\|q_{i}-q_{i}^{\prime}\right\|^{2} \\
c_{0} & =\frac{1}{20} \sqrt{w^{2}+h^{2}} \\
\sigma_{c}^{2} & =\frac{1}{10} \max _{m_{i}, m_{j}}\left\|c_{i}-c_{j}\right\|^{2} .
\end{aligned}
$$

The rationale is that we want high quality matches to be sampled with high

\begin{tabular}{|c|c|c|c|c|c|c|c|c|}
\hline & \multicolumn{4}{|c|}{$\begin{array}{c}\text { Synthetic single symmetry } \\
\text { LE [8] LHXS [6] CL [1] Proposed }\end{array}$} & \multicolumn{3}{|c|}{ | Synthetic multiple symmetries } & $\begin{array}{l}\text { nmetries } \\
\text { Proposec }\end{array}$ \\
\hline & $92 \%$ & $62 \%$ & $100 \%$ & $100 \%$ & $35 \%$ & & & \\
\hline & $12 \%$ & 070 & 15 & & $4 \%$ & $8 \%$ & 33 & \\
\hline & & & & & & & & \\
\hline $\mathrm{P} /$ & $\begin{array}{l}84 \% \\
68 \%\end{array}$ & $\begin{array}{c}29 \% \\
3 \%\end{array}$ & $\begin{array}{l}94 \% \\
69 \%\end{array}$ & $07 \Omega$ & $\begin{array}{l}43 \% \\
44 \%\end{array}$ & $\begin{array}{c}18 \% \\
0 \%\end{array}$ & $\begin{array}{l}68 \% \\
17 \%\end{array}$ & 16 \\
\hline
\end{tabular}
probability and that the second match is neither too close nor too far away from the first one.

\section{Experiments}

In all our experiments we fixed parameters $\delta=0.2$ and $\varepsilon=0.25$. We first tested the proposed method on the PSU dataset ${ }^{3}$. Performance is reported in Table 1.

Table 1. Performance comparison on the $\mathrm{PSU}$ dataset. $\mathrm{TP}=$ true positive, $\mathrm{FP}=$ false positive and GT=ground truth symmetries.

On synthetic datasets, the best performing method in the literature, due to Cho and Lee [1], detects all single symmetries, but also some erroneous ones ( $15 \%$ of false positives), while the proposed algorithm gives a perfect score. The result changes when multiple symmetries are present in the image. More of them are detected by the former method while our algorithm has a significantly lower false positive rate. On real images, the true positives are roughly the same for

\footnotetext{
${ }^{3}$ http://vision.cse.psu.edu/research/symmComp/
} 
both methods, but the false positives are smaller with our method when there is a single symmetry per image. In all cases, the method of Liu et al. [6] has a low false positive rate, but with a true positive rate that is well below the best performing methods. Figure 2 shows the results on some images of the dataset.

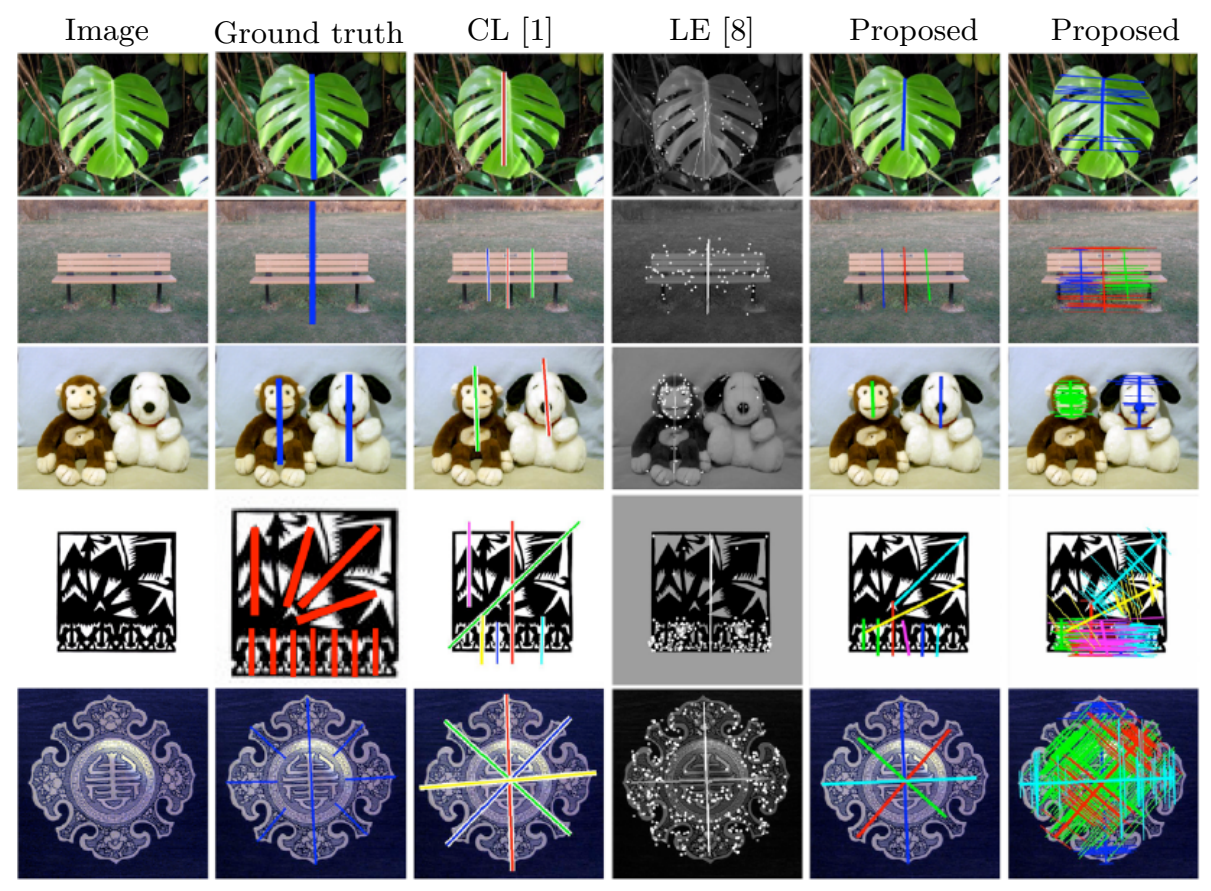

Fig. 2. Some examples of results from the PSU dataset. The last column shows the consensus set of matches for the detected symmetries, on top of the symmetry axes found by our method.

Additional results on other real images are shown in Figure 3. Notice that even with significant projective distortion, the symmetries are correctly detected by our method.

We applied the TILT algorithm of Zhang et al. [13] to some of the detected symmetry zones. This algorithm rectifies the zone by decomposing the intensity matrix into low-rank factors. Notice that this does not require the homography matrix $H$, which is not uniquely determined by the computation of $\hat{H}$ from (6) since the latest depends on only four parameters. Therefore a direct rectification of the image is not possible. 


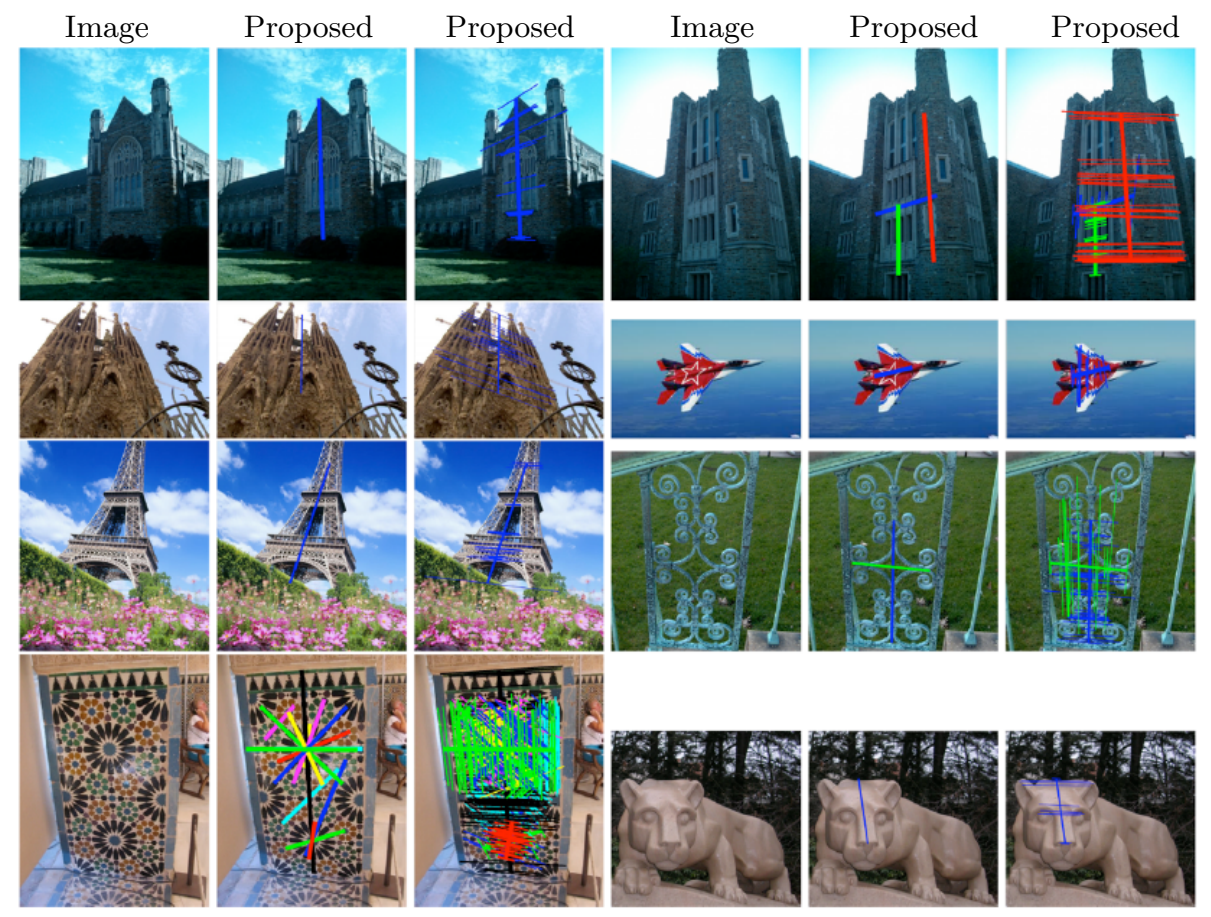

Fig. 3. Additional examples of our proposed method, with and without display of the supporting matches in addition of the symmetry axes.
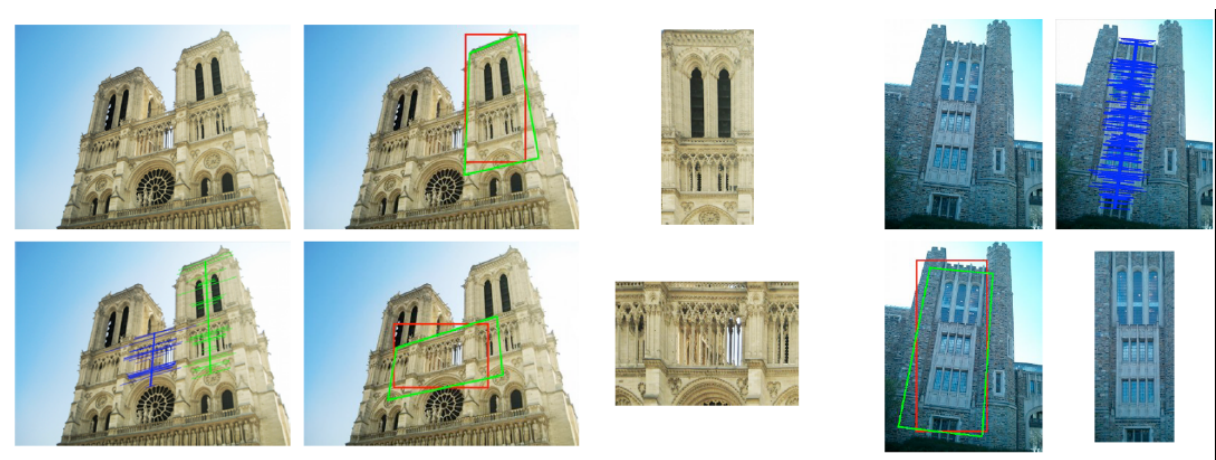

Fig. 4. Application of the TILT algorithm [13] to the zones of symmetry detected by our algorithm (in green boxes). The rectified zones are delimited by the red boxes. 


\section{Conclusion}

We have presented a new method for multiple symmetry detection in images. It relies on multiple model fitting through the J-linkage algorithm. We have demonstrated on experiments that the algorithm is able to detect symmetries with a moderate amount of perspective distortion. This ability could be extended with a more robust invariant feature matching algorithm, such as ASIFT [12]. The main drawback is the somewhat high false positive rate of detection, even if it is not worse than comparable detectors with the highest true positive rate. A way to control that would be to introduce an a contrario criterion [3] for validating a model into J-linkage. An alternative would be to use MAC-RANSAC [9]. Extensions to other Euclidean repetitions, like translation and rotation, are possible future work. Finally, an implementation in IPOL journal ${ }^{4}$ would be an interesting development.

\section{References}

1. Cho, M., Lee, K.M.: Bilateral symmetry detection via symmetry-growing. In: Proceedings of BMVC (2009)

2. Cox, D.A., Little, J., O'Shea, D.: Using algebraic geometry, vol. 185. Springer (2005)

3. Desolneux, A., Moisan, L., Morel, J.M.: From Gestalt theory to image analysis: a probabilistic approach, vol. 34. Springer (2007)

4. Fischler, M.A., Bolles, R.C.: Random sample consensus: a paradigm for model fitting with applications to image analysis and automated cartography. Communications of the ACM 24(6), 381-395 (1981)

5. Hartley, R., Zisserman, A.: Multiple view geometry in computer vision. Cambridge university press (2003)

6. Liu, Y., Hays, J., Xu, Y.Q., Shum, H.Y.: Digital papercutting. In: ACM SIGGRAPH 2005 Sketches. p. 99. ACM (2005)

7. Lowe, D.G.: Object recognition from local scale-invariant features. In: Computer vision, 1999. The proceedings of the seventh IEEE international conference on. vol. 2, pp. 1150-1157. IEEE (1999)

8. Loy, G., Eklundh, J.O.: Detecting symmetry and symmetric constellations of features. In: Computer Vision-ECCV 2006, pp. 508-521. Springer (2006)

9. Rabin, J., Delon, J., Gousseau, Y., Moisan, L.: MAC-RANSAC: a robust algorithm for the recognition of multiple objects. Proceedings of 3DPTV 2010 (2010)

10. Toldo, R., Fusiello, A.: Robust multiple structures estimation with J-linkage. In: Computer Vision-ECCV 2008, pp. 537-547. Springer (2008)

11. Tuytelaars, T., Turina, A., Van Gool, L.: Noncombinatorial detection of regular repetitions under perspective skew. Pattern Analysis and Machine Intelligence, IEEE Transactions on 25(4), 418-432 (2003)

12. Yu, G., Morel, J.M.: ASIFT: An algorithm for fully affine invariant comparison. Image Processing On Line 1 (2011), http://dx.doi.org/10.5201/ipol.2011. my-asift

13. Zhang, Z., Ganesh, A., Liang, X., Ma, Y.: TILT: transform invariant low-rank textures. International Journal of Computer Vision 99(1), 1-24 (2012)

\footnotetext{
$\overline{{ }^{4} \text { http://www } . i p o l . i m / ~}$
} 